\title{
Isomer yields in nuclear fission
}

\author{
A. Al-Adili ${ }^{1, *}, Z$. Gao ${ }^{1}, M$. Lantz ${ }^{1}, A$. Solders $^{1}, M$. Österlund ${ }^{1}$, and $S$. Pomp ${ }^{1}$ \\ ${ }^{1}$ Department of Physics and Astronomy, Uppsala University, Uppsala, Sweden
}

\begin{abstract}
The generation of angular momentum in the fission process is still an open question. To shed light on this topic, we started a series of measurements at the IGISOL-JYFLTRAP facility in Finland. High-precision measurements of isomeric yield ratios (IYR) are performed with a Penning trap, partly with the aim to extract average root-mean-square (rms) quantities of fragment spin distributions. The newly installed Phase-Imaging Ion-Cyclotron Resonance (PI-ICR) technique allows the separation of masses down to tens of $\mathrm{keV}$, which is sufficient to disentangle many isomers. In this paper, we first summarize the previous measurements on the neutron and proton-induced fission of uranium and thorium, e.g. the odd cadmium and indium isotopes $(119 \leq \mathrm{A} \leq 127)$. The measurements revealed systematic trends as function of mass number, which stimulated further exploration. A recent measurement was performed at IGISIOL and several new IYR data will soon be published, for the first time. Secondly, we employ the TALYS nuclear-reaction code to model one of the newly measured isomer yields. Detailed GEF and TALYS calculations are discussed for the fragment angular momentum distribution in ${ }^{134} \mathrm{I}$.
\end{abstract}

\section{Introduction}

While isomeric fission yields are an important part of a full fission-process description, the data libraries are incomplete and lack important systematic investigations of the isomer production cross section. Often isomeric ratio data are originating from $\gamma$-ray measurements, and there are wide discrepancies in the reported yields, most likely due to lack of nuclear structure data. Novel methods that reduce the dependence on level structures are important to improve the isomeric yield data situation. One example is the direct ion counting method used at IGISOL in Jyväskylä, Finland [1,2]. High precision mass measurements are performed using a Penning trap, which allows the mass separation of a meta-stable state from the ground state down to a mass difference of $50 \mathrm{keV} \mathrm{[3].}$

The fission fragments (FF) typically carry large angular momenta, $J$. The generation of $J$ still comprises challenges for contemporary fission modeling [4]. Many questions are unanswered, e.g. the conflicting experimental results concerning a possible saw-tooth trend in the average $J(A)$ [5]. Another important question is how angular momentum is shared among individual and collective degrees of freedom and how that eventually depends on the compound nucleus and excitation energy.

With the aid of nuclear de-excitation codes, one can model the angular momentum population of the primary fission fragments [6]. Albeit strongly model-dependent, this can shed

\footnotetext{
*e-mail: ali.al-adili@physics.uu.se
} 
some light on the systematics of angular momentum generation and the dependency on nuclear shell structure. The initial angular momentum possessed in the fragments will influence the number of emitted particles and their energy spectra. One such method was developed utilising the TALYS 1.9 reaction code [7]. Detailed description and benchmarking of this de-excitation model was published elsewhere [8].

\section{Summary of IYR experiments at IGISOL}

Fission fragments are produced by light charged particles accelerated up to tens of $\mathrm{MeV}$, that impinge on a fissionable target material [9]. Fission products stop in a helium-filled gas cell and are extracted for mass identification. Detailed simulations of the gas cell stopping efficiency and the associated systematic ion counting uncertainties can be found elsewhere [10]. A first isobaric selection $(A / q)$ is performed in a dipole magnet and a subsequent Paul trap cools and bunches the ions. The ion bunches are then injected into the Penning trap which consists of a purification trap and a precision trap [9].

Five IYR experiment campaigns have been performed at IGISOL within this collaboration, one on ${ }^{\text {nat }} U(n, f)$, three on ${ }^{\text {nat }} U(p, f)$ and one on ${ }^{232} \mathrm{Th}(\mathrm{p}, \mathrm{f})$. Three different methods have been utilized for particle identification and counting: $\gamma$-ray spectroscopy, side-band cooling technique [9] and Phase-Imaging Ion-Cyclotron Resonance (PI-ICR) technique [11]. A summary of the measurements and the associated analysis methods is extensively covered elsewhere $[12,13]$. Here we review the most important findings from each measurement campaign.

\subsection{IYRs from ${ }^{\text {nat }} U(\mathbf{n}, \mathbf{f})$}

The first isomeric yield ratios measured within the Uppsala-Jyväskylä collaboration was based on $\gamma$-ray spectroscopy using High-Purity Germanium detectors. Yield ratios of three tin isotopes (cumulative) and two antimony isotopes (independent) were measured in the ${ }^{n a t} \mathrm{U}(\mathrm{n}, \mathrm{f})$ reaction with neutron energies of $E_{\mathrm{n}}=12.4 \pm 8.8 \mathrm{MeV}[12,14]$. The results were suffering from rather large uncertainties due to low neutron production, but still revealed some interesting differences to evaluated ENDF B-VII.1 files.

\subsection{IYRs from ${ }^{\text {nat }} \mathrm{U}(\mathrm{p}, \mathrm{f})$ and ${ }^{232} \mathrm{Th}(\mathrm{p}, \mathrm{f})$ with Side-band cooling}

In the second campaign the focus was put on using the Penning trap and to measure several new isomeric yields using the so-called side band cooling technique [1]. The studied reactions were ${ }^{\text {nat }} \mathrm{U}(\mathrm{p}, \mathrm{f})$ and ${ }^{232} \mathrm{Th}(\mathrm{p}, \mathrm{f})$ with $E_{\mathrm{p}}=25.0 \mathrm{MeV}$. Results for six fission products (three from the light fragment peak and three from the heavy peak) were reported [1]. An example is shown in Fig. 1 for ${ }^{81} \mathrm{Ge}$. The plot shows a frequency scan in the Penning trap, which upon matching a characterising resonance frequency, filters the ions (based on mass). Differences between the two fissioning systems were observed, hinting to differences in populated angular momenta. An attempt to extract angular momentum root-mean-square (rms) values $J_{\text {rms }}$ was done. Most deduced values turned out to be much larger than what is observed in thermal reactions, hinting to a larger angular momentum population in the compound nucleus at higher excitation energies [15].

\subsection{IYRs from nat $U(p, f)$ with PI-ICR}

The side-band cooling technique has a mass resolution limited to about half an $\mathrm{MeV}$, which is illustrated in the example of ${ }^{81} \mathrm{Ge}$ (Fig. 1). The mass difference between ground- and excited 


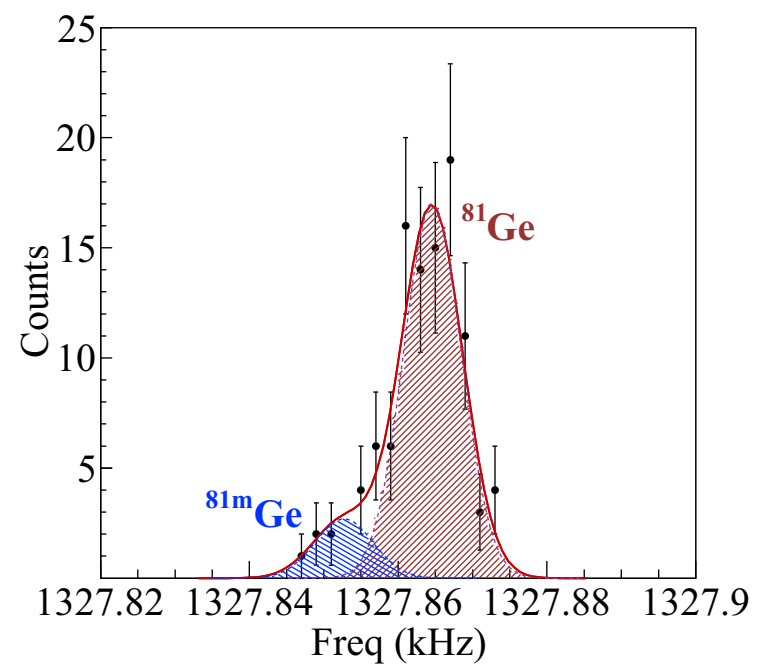

Figure 1. The IYR of ${ }^{81} \mathrm{Ge}$ extracted through Gaussian fits to the isomer $(E=679 \mathrm{keV})$ and the ground state [1]. The data were obtained using the side-band cooling technique, which has a mass resolution limit of about $500 \mathrm{keV}$ for isomer separation.

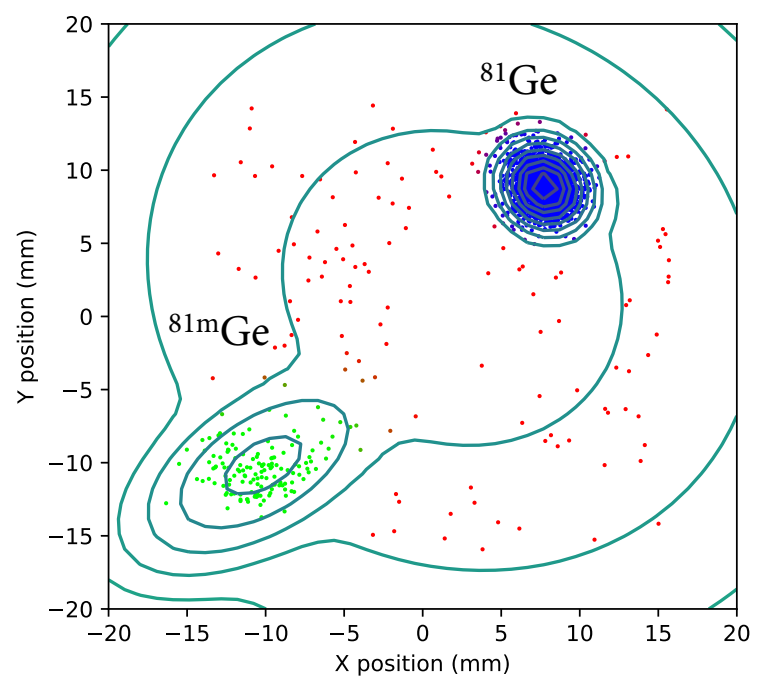

Figure 2. An example from ${ }^{81} \mathrm{Ge}$, where the Phase-Imaging Ion-Cyclotron Resonance (PI-ICR) technique was employed. The plot illustrates the X,Y coordinates of a position-sensitive Micro-Channel Plate. The ground state is well separated from the meta-stable state.

states in ${ }^{81} \mathrm{Ge}$ is $679 \mathrm{keV}$, which causes the peaks to overlap. This leads to growing uncertainties in the deduced IYR. With the birth of the novel PI-ICR technique [3], the achieved mass resolution is about 10 times better, proving the ${ }^{81} \mathrm{Ge}$ isomers easy to disentangle as seen in Fig. 2 [13]. A two-dimensional fit is performed using Bayesian mixing which yields the weight of each isotope by calculating the probability of each data point belonging to any of the peaks. 
The new PI-ICR technique has been used in two measurement campaigns on ${ }^{\text {nat }} \mathrm{U}(\mathrm{p}, \mathrm{f})$ and with $25 \mathrm{MeV}$ protons. A systematic study was made on 10 new isomeric yields, five odd-A cadmium isotopes and an equal number of odd-A indium isotopes, all produced in the fission of ${ }^{\text {nat }} \mathrm{U}(\mathrm{p}, \mathrm{f})$. The data revealed a decreasing trend of high-spin state population as a function of mass for indium, and an increasing trend for cadmium [2]. After calculating the root-mean-square of $J$, a correlation was found with the nuclear quadrupole moments. A possible explanation could be a change in the fragment shape which causes the angular momentum to change, because of varying moment of inertia.

A second campaign was recently completed and 18 new isomeric ratios were measured, close to shell closure in the Sn-132 region. A total of about 30 isomers have now been measured, with the aim to investigate how shell effects influence the IYR and the angular momentum population. Analysis of the most recent data is still ongoing. Below we show detailed de-excitation calculations for one of the measured isotopes, ${ }^{134} \mathrm{I}$, to demonstrate the most recent development in our GEF+TALYS-based method. A simplified decay scheme is depicted in Fig. 3 which involves the lower discrete levels including the meta-stable state $\left(8^{-}\right)$ and the ground state $\left(4^{+}\right)$.

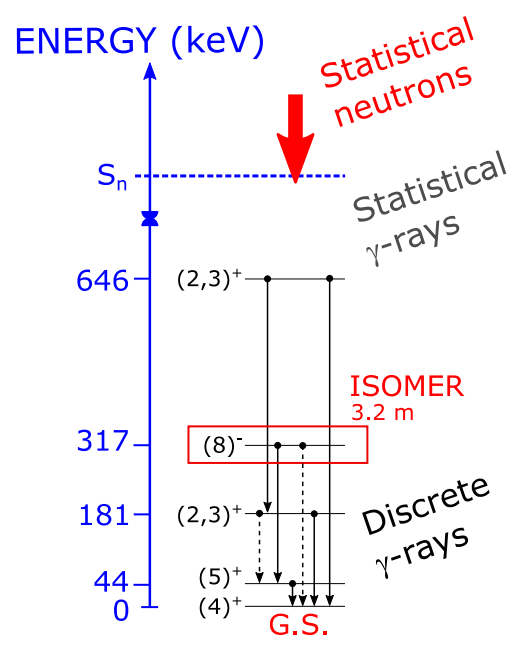

${ }^{134} \mathrm{I}$

Figure 3. A simplified decay scheme of ${ }^{134} \mathrm{I}$. The isomeric state has an energy of $317 \mathrm{keV}$ and decays by internal transition.

\section{Model calculations for ${ }^{134}$ I}

TALYS uses the statistical Hauser-Feshbach evaporation model which takes into account the competition between neutrons and $\gamma$-rays, in addition to the RIPL-3 data-base for discrete levels. We have used the Microscopic-Goriely level density model and up to 10 discrete levels for each nucleus. In order to perform the calculations, one needs the initial parameterisation of excitation energy and angular momentum. The spin distribution is assumed to follow the commonly used level density function, based on a Rayleigh probability distribution:

$$
P(J) \propto(2 J+1) \exp \left(-\frac{(J+0.5)^{2}}{B^{2}}\right) .
$$




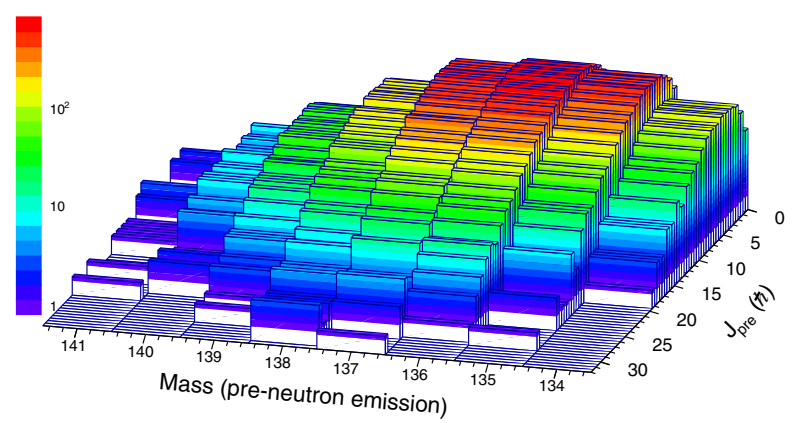

Figure 4. The angular momentum distributions obtained with GEF, for each fission fragment leading to ${ }^{134}$ I as fission product after neutron emission. Each fission fragment case was modelled in TALYS for different values of $B$ (see Eq. (1)).
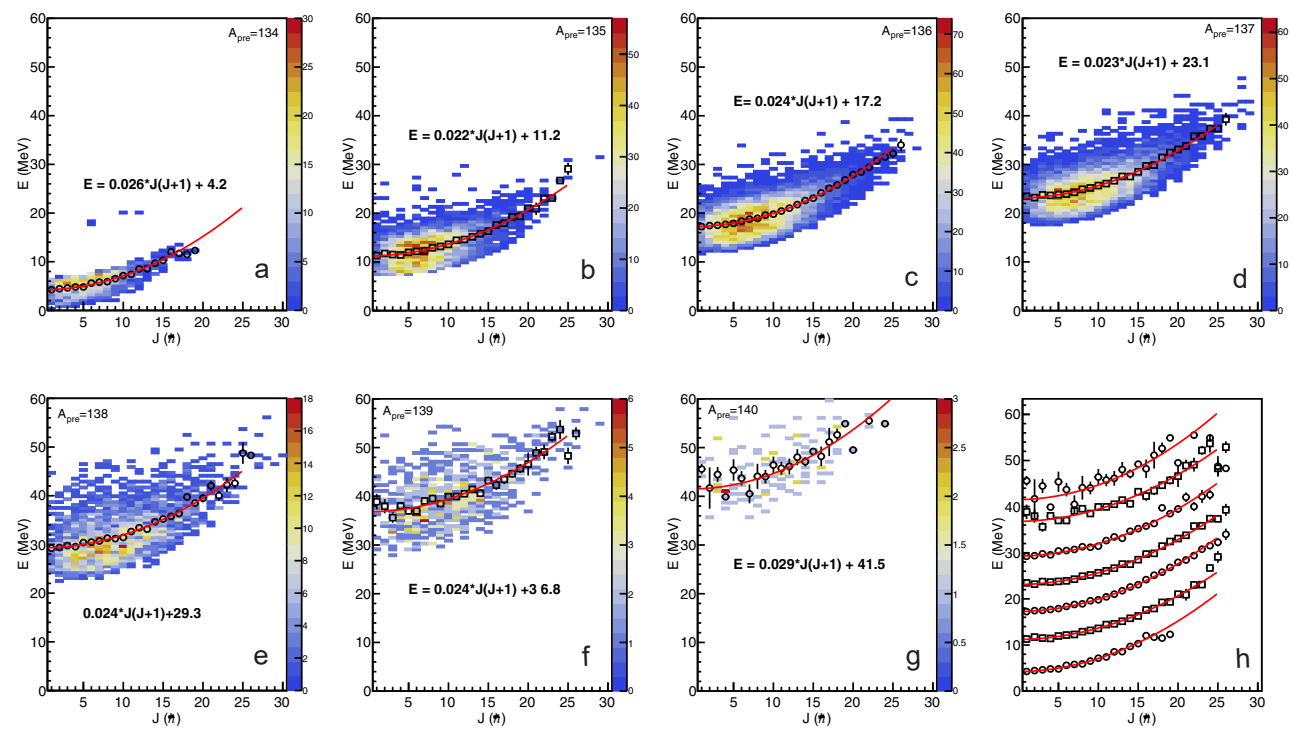

Figure 5. The excitation energy, $E$, versus spin, $J$, for all fission fragments leading to ${ }^{134} \mathrm{I}$, as simulated with GEF. The average energies are parameterised by Eq. (2) with $C_{1}$ and $C_{2}$ acting as free fit parameters. Plot $\mathrm{h}$ shows all the fits from a-g.

The "spin cut-off" parameter $B$, defines the mean and width of the distribution and is normally approximated with the root-mean-square, $J_{\mathrm{rms}}$, of $P(J)$. By varying $B$, it is possible to obtain the value that reproduces the experimental result [8]. In this work we use the prediction IYR value of GEF as the benchmark for these calculations.

The angular momentum distributions were simulated using the GEF code (V.2017-1.2) [16], for all fragments leading to ${ }^{134}$ I after neutron emission (Fig. 4). Up to 7 neutrons may be emitted, all with a unique $P(J)$ distribution. Figure 5 illustrates the excitation energy versus spin for each fission fragment leading to ${ }^{134} \mathrm{I}$. The average excitation energy, $\bar{E}$, increases as 

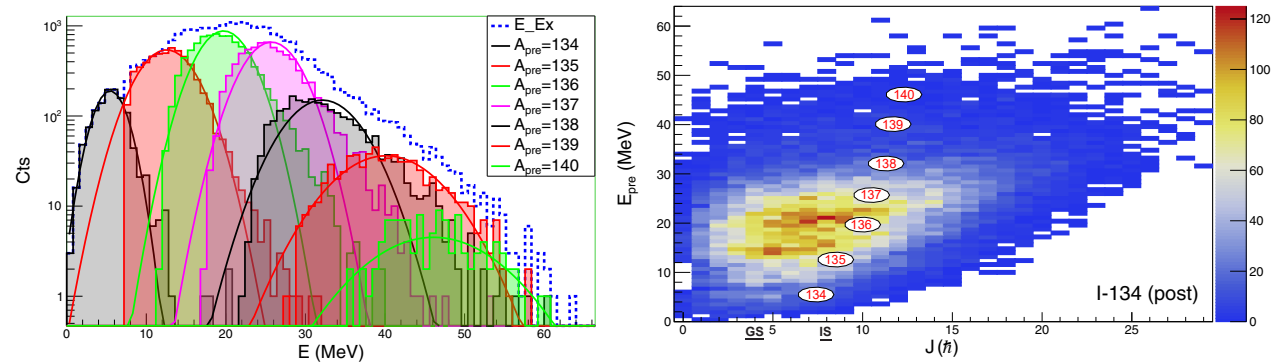

Figure 6. Left: the excitation energy distribution for each fission fragment leading to ${ }^{134} \mathrm{I}$ after neutron emission. Normal distributions were fitted to each distribution to retrieve average energies and spreads $\sigma_{\mathrm{E}}$. Right: the spin versus $E^{*}$ distribution for all distributions in Fig. 5, superimposed. The spin values of ground state and meta-stable state are indicated on the spin axis. The average excitation energies (left plot of this figure) and $J_{\mathrm{rms}}$ values are indicated for each individual fragment by numbered circles. The total GEF $P(J)$ distribution has a $J_{\text {rms }}$ value of $9.5 \hbar$.

function of fragment spin $J$ due to the increase in rotational energy:

$$
\bar{E}(J)=C_{1}\left(J^{2}+J\right)+C_{2} \propto \frac{\hbar^{2}}{2 \mathcal{J}} J(J+1) .
$$

where $\mathcal{J}$ is the moment of inertia. $C_{1}$ and $C_{2}$ act as free fit parameters. The coefficient $C_{1}$ seems to be independent of the parent mass while $C_{2}$ depends on the neutron separation energy.

In this work, we provide TALYS with input files parameterising the excitation energy with Gaussian distributions, which is a good first order approximation for each neutron emission channel. We deduce the average energy and spread of energy $\left(\sigma_{\mathrm{E}}\right)$ from each Gaussian fit as seen in Fig. 6 (left). More refined excitation energy parameterisation is currently under development, where the functional dependencies of Eq. (2) and Fig. 5 will be implemented.

The superposition of all the neutron channels is plotted in Fig. 6 (right) together with the respective average energies (from the Gaussian fits) and $J_{\mathrm{rms}}$ as indicated by numbered circles (mass number, $A$ ). The total angular momentum distribution from GEF, summed over all fission fragments, has a $J_{\text {rms }}$ of $9.5 \hbar$. As expected, there is a positive correlation between the average energy and the average spin [13].

All in all, 56 TALYS IYR calculations were performed (eight spin distributions for each of the seven pre-cursors), all of which are plotted in Fig. 7. The IYR is defined as the ratio of high-spin cross section to the total production cross section. As the $B$ parameter increases, higher spin values are populated by the de-exciting nucleus. Therefore, the population of the high-spin state will increase with $B$. The vertical lines correspond to $J_{\text {rms }}$ as extracted from the GEF distributions plotted in Fig. 4. GEF predicts an IYR of about 0.55. Based on this value the average $J_{\text {rms }}$ from TALYS is between 9.5 and $10.5 \hbar$, for the case of ${ }^{134} \mathrm{I}$.

\section{Conclusions and outlook}

With the different techniques employed, we have thus far measured IYR for 34 different products for three different reactions at the IGISOL facility in Finland [1, 2, 14]. The bulk of the data, 30 IYR, are in the ${ }^{132} \mathrm{Sn}$ region and for ${ }^{238} \mathrm{U}(\mathrm{p}, \mathrm{f})$ at $25 \mathrm{MeV}$. The analysis carried out on the data this far have already revealed interesting trends. It remains to be seen what can be learned from the most recent data-set, offering to study the behaviour across the $N=82$ line 


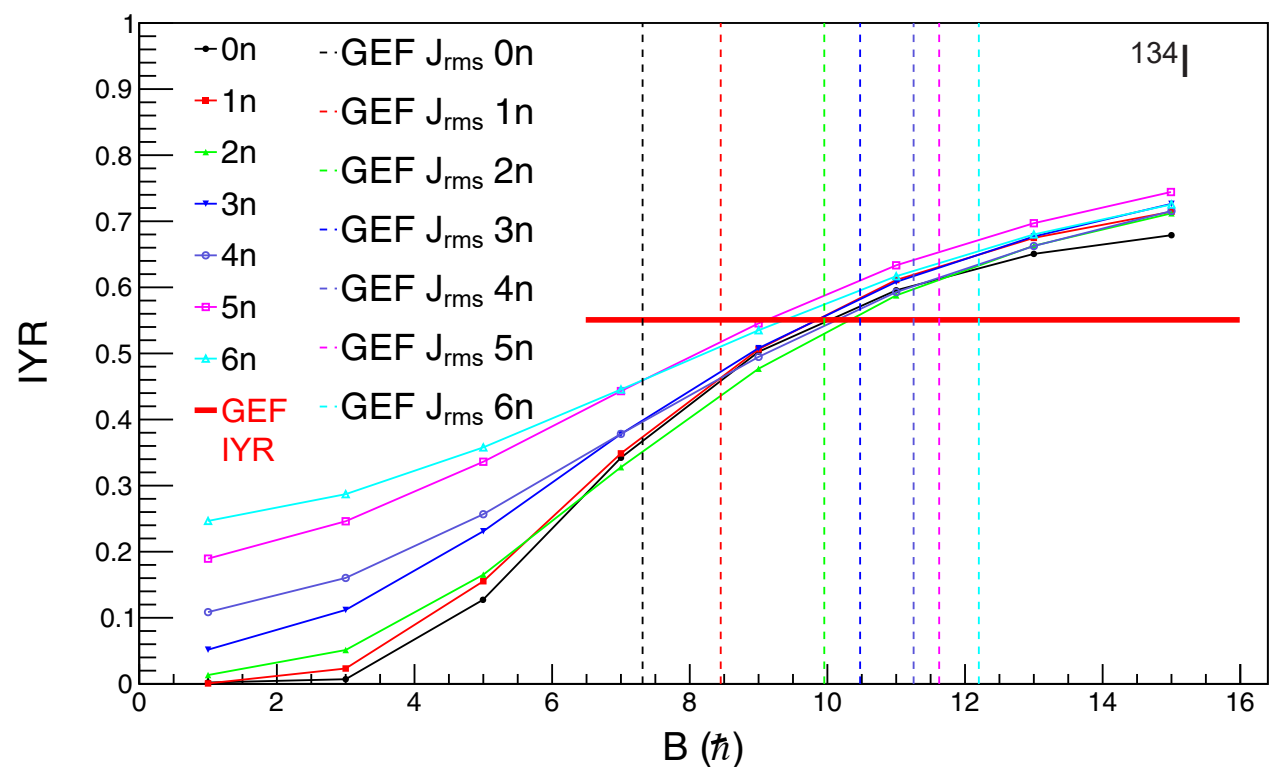

Figure 7. TALYS and GEF calculations for the isomeric yield ratio of ${ }^{134} \mathrm{I}$, for all fragments $(134 \leq A \leq 140 \mathrm{u})$ giving rise to ${ }^{134} \mathrm{I}$, after prompt neutron emission. The deduced GEF $J_{\text {rms }}$ values are indicated by the vertical lines for each neutron channel. GEF gives a total of $9.5 \hbar$ as an average of all channels and the TALYS calculations point to a $J_{\text {rms }}$ value between 9.5 and $10 \hbar$.

for three different elements, as well as trends along $N=81$. There are several different options that seem worth exploring and that will strengthen the experimental database to improve our understanding of the fission process and to develop and constrain fission model codes.

One option is to study the obtained cadmium and indium behavior of the IYR in the ${ }^{238} \mathrm{U}(\mathrm{n}, \mathrm{f})$ case at lower excitation energies to allow unfolding the contributions from different fission systems by reducing the contributions from higher fission chances. Measuring at, say, $12 \mathrm{MeV}$, also offers the chance to see possible effects from the angular momentum of the compound system.

Another option is to expand the studies to other regions of the nuclear chart. Especially interesting would be to look at the ${ }^{78} \mathrm{Ni}$ region and to explore complementary fission fragments. While the technique does not allow for coincidence measurements, it might be fruitful to explore trends along complementary nuclear charges, e.g., compare the trends seen for In nuclei $(Z=49)$, with trends at $Z=44(\mathrm{Tc})$ in the $\mathrm{U}(\mathrm{p}, \mathrm{f})$ case, or $Z=42$ (Mo) for proton-induced-fission of thorium.

Thirdly, it is of course desirable to expand the IYR database for the two systems were we thus far only scratched the surface, as well as studying other systems. Particularly interesting would be the case of spontaneous fission, e.g., of ${ }^{248} \mathrm{Cm}$ or ${ }^{252} \mathrm{Cf}$, without the need for an accelerator.

To achieve these ends, there are some possible developments at the IGISOL facility. A development of new ion guides are important, where we try to increase stopping efficiencies and decrease extraction times. This could facilitate more measurements of IYR in neutroninduced fission.

Using other measurement techniques to measure IYR that are not measurable at JYFLTRAP, should also be looked at. While the PI-ICR technique offers excellent mass resolving power (MRP), MR-TOF systems allow for faster measuring times. Thus, isotopes 
with shorter half-lives as well as lower yield regions can be targeted. One option for this is the use of newly implemented MR-TOF system in Jyväskylä. Another possibility is the use of the MR-TOF system at the Cryogenic Stopping Cell (CSC) at GSI. The MR-TOF technique has recently showed significant improvement in achievable MRP, which reached a value of $10^{6}$ at GSI [17].

We also consider using the more standard $\gamma$-measurement techniques for cases of very short half-lives or low excitation energies, not accessible with the above techniques, but of importance for our study. Following along this research track should result in a rich database of IYR for various cases, and provide insight in the physics behind the generation of the large fragment angular momenta in the fission process.

Finally, we envisage further development of the TALYS-driven model through collaboration with the code developers at the IAEA. Plans for further verification and benchmarking are ongoing, especially to understand how other fission reaction mechanisms (e.g. Coulomb excitation, abrasion fission and fusion-fission) affect the de-excitation of fragments and the isomer population.

Funding for this work was received from the European Commission within the CHANDA project (contract 605203), the Swedish Radiation Safety Authority (SSM) and the Swedish Research Council (VR).

\section{References}

[1] V. Rakopoulos, M. Lantz, A. Solders, A. Al-Adili, A. Mattera, L. Canete, T. Eronen, D. Gorelov, A. Jokinen, A. Kankainen et al., Phys. Rev. C 98, 024612 (2018)

[2] V. Rakopoulos, M. Lantz, S. Pomp, A. Solders, A. Al-Adili, L. Canete, T. Eronen, A. Jokinen, A. Kankainen, A. Mattera et al., Phys. Rev. C 99, 014617 (2019)

[3] S. Eliseev, K. Blaum, M. Block, C. Droese, M. Goncharov, E. Minaya Ramirez, D.A. Nesterenko, Y.N. Novikov, L. Schweikhard, Phys. Rev. Lett. 110, 082501 (2013)

[4] A.N. Andreyev, K. Nishio, K.H. Schmidt, Rep. Prog. Phys. 81, 016301 (2018)

[5] C. Wagemans, The nuclear fission process (CRC Press, 1991), ISBN $978-0849354342$

[6] S. Okumura, T. Kawano, P. Jaffke, P. Talou, S. Chiba, J. Nucl. Sci. Technol. 55, 1009 (2018)

[7] A. Koning, D. Rochman, S. van der Marck, Nucl. Data Sheets 118, 187 (2014)

[8] Al-Adili, A., Rakopoulos, V., Solders, A., Eur. Phys. J. A 55, 61 (2019)

[9] H. Penttilä, P. Karvonen, T. Eronen, V.V. Elomaa, U. Hager, J. Hakala, A. Jokinen, A. Kankainen, I.D. Moore, K. Peräjärvi et al., Eur. Phys. J. A 44, 147 (2010)

[10] A. Al-Adili, K. Jansson, M. Lantz, A. Solders, D. Gorelov, C. Gustavsson, A. Mattera, I. Moore, A.V. Prokofiev, V. Rakopoulos et al., Eur. Phys. J. A 51, 59 (2015)

[11] D.A. Nesterenko, T. Eronen, A. Kankainen, L. Canete, A. Jokinen, I.D. Moore, H. Penttilä, S. Rinta-Antila, A. de Roubin, M. Vilen, Eur. Phys. J. A 54, 154 (2018)

[12] A. Mattera, Ph.D. thesis, Uppsala University, Applied Nuclear Physics (2017)

[13] V. Rakopoulos, Ph.D. thesis, Uppsala University, Applied Nuclear Physics (2018)

[14] A. Mattera, S. Pomp, M. Lantz, V. Rakopoulos, A. Solders, A. Al-Adili, H. Penttilä, I. D. Moore, S. Rinta-Antila, T. Eronen et al., Eur. Phys. J. A 54, 33 (2018)

[15] D.C. Aumann, W. Gückel, E. Nirschl, H. Zeising, Phys. Rev. C 16, 254 (1977)

[16] K.H. Schmidt, B. Jurado, C. Amouroux, C. Schmitt, Nucl. Data Sheets 131, 107 (2016), special Issue on Nuclear Reaction Data

[17] T. Dickel, Priv. Comm. (2019) 